\title{
Local insulin-like growth factor I expression is essential for Purkinje neuron survival at birth
}

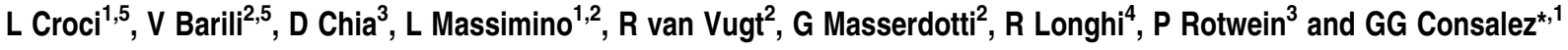

IGF1, an anabolic and neuroprotective factor, promotes neuronal survival by blocking apoptosis. It is released into the bloodstream by the liver, or synthesized locally by muscles and neural cells, acting in an autocrine or paracrine fashion. Intriguingly, genetic studies conducted in invertebrate and murine models also suggest that an excess of IGF1 signaling may trigger neurodegeneration. This emphasizes the importance of gaining a better understanding of the mechanisms controlling IGF1 regulation and gene transcription. In the cerebellum, Igf1 expression is activated just before birth in a subset of Purkinje cells (PCs). Mice carrying a null mutation for HLH transcription factor EBF2 feature PC apoptosis at birth. We show that lgf1 is sharply downregulated in Ebf2 null PCs starting before the onset of PC death. In vitro, EBF2 binds a conserved distal Igf1 promoter region. The pro-survival PI3K signaling pathway is strongly inhibited in mutant cerebella. Finally, Ebf2 null organotypic cultures respond to IGF1 treatment by inhibiting PC apoptosis. Consistently, wild type slices treated with an IGF1 competitor feature a sharp increase in PC death. Our findings reveal that IGF1 is required for PC survival in the neonatal cerebellum, and identify a new mechanism regulating its local production in the CNS.

Cell Death and Differentiation (2011) 18, 48-59; doi:10.1038/cdd.2010.78; published online 2 July 2010

A large body of evidence shows that programmed cell death is required for the appropriate completion of neural development in many species, as exemplified by apoptosis of Rohon Beard primary sensory neurons in Xenopus embryos at metamorphosis, ${ }^{1}$ and by the dramatic effects of Apaf1 mutation in mice. ${ }^{2}$ In the developing mouse cerebellum, a considerable percentage of PC progenitors born in the ventricular zone undergoes apoptosis; accordingly, in transgenics overexpressing the anti-apoptotic factor $B C l 2, P C s$ are substantially increased in number. ${ }^{3}$

During development, many neural cells are programmed to die, whereas others make use of antiapoptotic factors for their survival. Some of these are target-derived, while others act anterogradely. A large collection of secreted proteins, ranging from neurotrophins to various classes of trophic factors and cytokines, has been found to promote neuronal survival in development. ${ }^{4}$

Several authors have shown that, in many of the mouse mutants in which Purkinje neurons are affected, cell death often follows precise patterns that recapitulate the subdivision of these neurons into metabolically and molecularly defined subgroups (reviewed in Dusart et al..$^{5}$ ). In cerebellar mutants, PC death can take different forms, exhibiting mixed features of apoptosis, necrosis and autophagy. For instance, in Lurcher, a gain-of-function mutation for the delta 2 glutamate receptor, PC death occurs through a variety of cellular processes, ${ }^{6}$ including autophagy.

One of the questions remaining to be addressed in the field is the transcriptional regulation of neuronal survival. Not only is this relevant to our understanding of normal neural development, but it would assist us in dissecting the nature of repair processes that are reactivated or repressed in response to noxious stimuli. Our group and others have analyzed the roles of Collier/Olf/Ebf transcription factors (TFs) in neural development. Ebf genes (reviewed in Dubois and Vincent ${ }^{7}$ ), encode helix-loop-helix TFs highly conserved in evolution, that were originally characterized for their roles in the immune system, and subsequently implicated in various aspects of neural development, including neuronal differentiation, ${ }^{8}$ migration and axon fasciculation and guidance (inter alia Garel et al. ${ }^{9}$ ). One member of this family, Ebf2, has relevant roles in neuroendocrine, olfactory and peripheral nerve development. ${ }^{10,11}$ We and others have shown that EBF2 is important for cerebellar cortical patterning ${ }^{12,13}$ and PC survival. On postnatal day 1, Ebf2 null mutants lose $38 \%$ of their PCs, due to apoptotic cell death. ${ }^{12}$ This observation prompted us to search for EBF2 targets involved in PC survival during postnatal development.

Abundant evidence supports a role for Insulin-like growth factor 1 and 2 (IGF1 and IGF2) in central nervous system (CNS) development. IGF1 is predominantly expressed in neurons in a fashion that coincides with outbursts of neural progenitor proliferation and/or neurite outgrowth. In contrast, IGF2 expression becomes confined mainly to cells of mesenchymal and neural crest origin. IGF1R is broadly expressed, while IGF-binding proteins are regionally and developmentally restricted, correlating with peaks of IGF expression (reviewed in D'Ercole et al. ${ }^{14}$ ). In the rodent

\footnotetext{
${ }^{1}$ Division of Neuroscience, San Raffaele Scientific Institute, Milan, Italy; ${ }^{2}$ Vita-Salute San Raffaele University, Milan, Italy; ${ }^{3}$ SOM-Biochemistry \& Molecular Biology Department, Oregon Health and Science University, Portland, OR, USA and ${ }^{4}$ ICRM, Consiglio Nazionale delle Ricerche, Milan, Italy

*Corresponding author: GG Consalez, Division of Neuroscience, San Raffaele Scientific Institute, Via Olgettina 58, 20132 Milano, Italy. Tel: + 3902 2643 4838 ;

Fax: + 39022643 5283; E-mail: g.consalez@hsr.it

${ }^{5}$ These authors contributed equally to this work.

Keywords: Purkinje cells; IGF1; EBF2; transcription; apoptosis; ataxia

Abbreviations: AKT/PKB, protein kinase B; EBF2, early B-cell factor 2; IGF1, insulin-like growth factor I; PCs, Purkinje cells; PI3K, phosphatidylinositol 3-kinase; $\mathrm{TF}$, trancription factor

Received 03.3.10; revised 29.4.10; accepted 13.5.10; Edited by L Greene; published online 02.7.10
} 
cerebellum, PCs are the main expression site of IGF1, starting around birth and declining at about P20, to continue throughout adulthood, ${ }^{15}$ and transgenic overexpression of Igf1 has been shown to promote cerebellar cell survival. ${ }^{16}$

In this paper, we identify Igf1 as a potential transcriptional target of EBF2 using a computer-based prediction approach of EBF target site-containing genes. We show that a subset of Ebf2-positive PCs expresses the Igf1 gene, and that Igf1 expression is profoundly downregulated in Ebf2 null PCs, which die by classical apoptosis. Furthermore, IGF1/PI3K signaling is downregulated in Ebf2 null cerebella at birth, and the addition of an IGF1 inhibitor triggers PC death in wild-type (wt) slices at the same stage, whereas treatment with recombinant IGF1 of Ebf2 null organotypic cultures rescues $\mathrm{PC}$ death. Our results reveal that IGF1 is strictly required for PC survival at birth and shed new light on the local control of Igf1 gene expression in neurons. This may have farther reaching implications for the study of molecular mechanisms regulating neuronal survival and degeneration.

\section{Results}

Ebf2 null PCs die by apoptosis within $24 \mathrm{~h}$ of birth. At birth, Ebf2 null cerebella feature a marked increase in the number of activated caspase 3 (AC3) + apoptotic bodies in the PC layer, particularly in the anterior lobe, that mainly harbors Ebf2+ , zebrinll- PCs. ${ }^{12}$ At this stage, AC3 staining in the PC layer colocalizes with the PC markers retinoic acidrelated orphan receptor $\alpha(\mathrm{ROR} \alpha)$ (Figure 1a-c) or calbindin (CaBP) (Figure 1d and Croci et al. ${ }^{12}$ ). PC death in the cerebellum, described extensively in wt and mutant mice, follows at least three different avenues: necrosis, classical apoptosis and dysregulated autophagy (reviewed in Dusart et al. ${ }^{5}$ ).

To better define the type of death affecting Ebf2 null PCs, we analyzed mutant and wt cerebella at postnatal day 0.5 (P0.5) using a combined approach: at first, we applied terminal deoxynucleotidyl transferase dUTP nick-end labeling (TUNEL) staining, detecting nuclear DNA fragmentation. TUNEL + cells in the cerebellum were counted as described in Materials and Methods. Our results indicate that, at P0.5, both wt and null cerebella feature a relevant and equivalent degree of TUNEL positivity ( $P=0.51$, Student's $t$-test) in the external granular layer. However, only mutant cerebella feature numerous TUNEL + nuclei in the PC layer $(P<0.001)$ (arrows in Figure $1 f$ and h, Supplementary Figure 1D) and a significant increase in cell death $(P<0.01)$ in the prospective internal granule layer (IGL) and white matter (WM), which, at this stage, hosts late-migrating PCs $(\mathrm{CaBP}+)$, oligodendrocyte progenitors (Olig2 +), GABA interneurons $($ Pax $2+)$, few early migrating granule cells and unipolar brush cells (both Pax6 + ) (reviewed in Carletti and Rossi ${ }^{17}$ ). By immunofluorescence, we found that the majority of apoptotic bodies are $\mathrm{CaBP}+{ }^{12}$ (Figure 1d, arrows), whereas only a low percentage expresses Pax2, Pax6 or Olig2 (Figure 1i-k, arrows).

To confirm that mutant PCs die by apoptosis, we analyzed Ebf2 null cerebella by electron microscopy. Our results show that the mutant PC layer contains apoptotic bodies characterized by pycnotic, electron-dense nuclei and condensed cytoplasms (Figure $1 \mathrm{~m}$ and o). Such cellular phenotypes are not detected in the cerebellum of littermate controls (Figure $1 \mathrm{l}$ and $\mathrm{n}$ ).

In organotypic cerebellar slices and in the wt cerebellum, PC death occurs principally between E15 and P6 (reviewed in Dusart et al. ${ }^{5}$ ). We analyzed the cerebellum of Ebf2 nulls and controls at E19.5 (Supplementary Figure $1 A$ and B), P3 (Supplementary Figure $1 E$ and F) and P7 (not shown). Our results clearly indicate that the frequency of AC3 and TUNEL + PCs in Ebf2 null cerebella is comparable to wt levels at all stages, while it is increased severely and selectively right after birth (Supplementary Figure 1C and D).

Identification of Igf1 as a candidate EBF2 target gene. In the newborn cerebellum, Ebf2, as shown by $\beta$-galactosidase immunofluorescence in $E b f 2^{+/ L a c Z}$ mice, ${ }^{10}$ is expressed specifically in PCs, where it colocalizes with CaBP (Figure $2 a$ and b). Since EBF2 is a transcription factor (TF), ${ }^{18}$ we interrogated sequence databases for genes carrying putative EBF target site $(\mathrm{s})^{19}$ in their $5^{\prime}$ flanking region, and encoding factors implicated in survival. To this end, we used the Targetfinder software. ${ }^{20}$ One potentially interesting hit was Igf1, that encodes a secreted survival factor. ${ }^{14}$ An evolutionary analysis revealed that two putative EBFbinding sites lie in a conserved lgf1 promoter region (Supplementary Figure 2) (EBF-A and $E B F-B$, see Materials and Methods), and one of them $(E B F-B)$ is conserved from mouse to human.

Based on this evidence, we compared the expression of EBF2 and IGF1 in the cerebellum. Ebf2 and Igf1 colocalize in PCs at E19.5 (Supplementary Figure 3) and P0.5 (Figure 2c). To determine whether IGF1 protein is also expressed in PCs, we performed immunofluorescence staining using IGF1 and CaBP antisera. Our results show that many PCs express IGF1 at birth (Figure 2d, higher magnification in panel e). At the same stage, IGF1 is not expressed in any other components of the cerebellar cortex. This result, along with that shown in Figure $2 a$ and $b$, demonstrates that IGF1 and EBF2 are co-expressed in neonatal PCs.

Downregulation of IGF1 gene and protein in Ebf2 null PCs. In light of the above, we asked if Igf1 expression is downregulated in Ebf2 null PCs either before or after birth. As shown by in situ hybridization, Igf1 transcript levels are sharply decreased in null PCs at both stages (Figure $3 b$ and $\mathrm{d}$ ). We also analyzed protein lysates from wt and null cerebella ( $n=3$ per genotype). Results are shown in Figure $3 e$ and plotted in Figure $3 f$. Taken together, these data indicate that, in Ebf2 null cerebella, IGF1 is downregulated both at the transcript and protein level.

EBF2 regulates Igf1 gene expression in cultured neural cell lines. Subsequently, we set out to confirm the potential transcriptional regulation of Igf1 expression by EBF2. To this end, we moved to cultured P19 cells, a multipotent mouse embryo-derived teratocarcinoma cell line. These cells, upon retinoic acid (RA) treatment, neuralize and activate Ebf2 expression (G. Masserdotti and G.G. Consalez, unpublished). By reverse transcription-quantitative polymerase chain reaction (RT-qPCR), we analyzed lgf1 expression after 

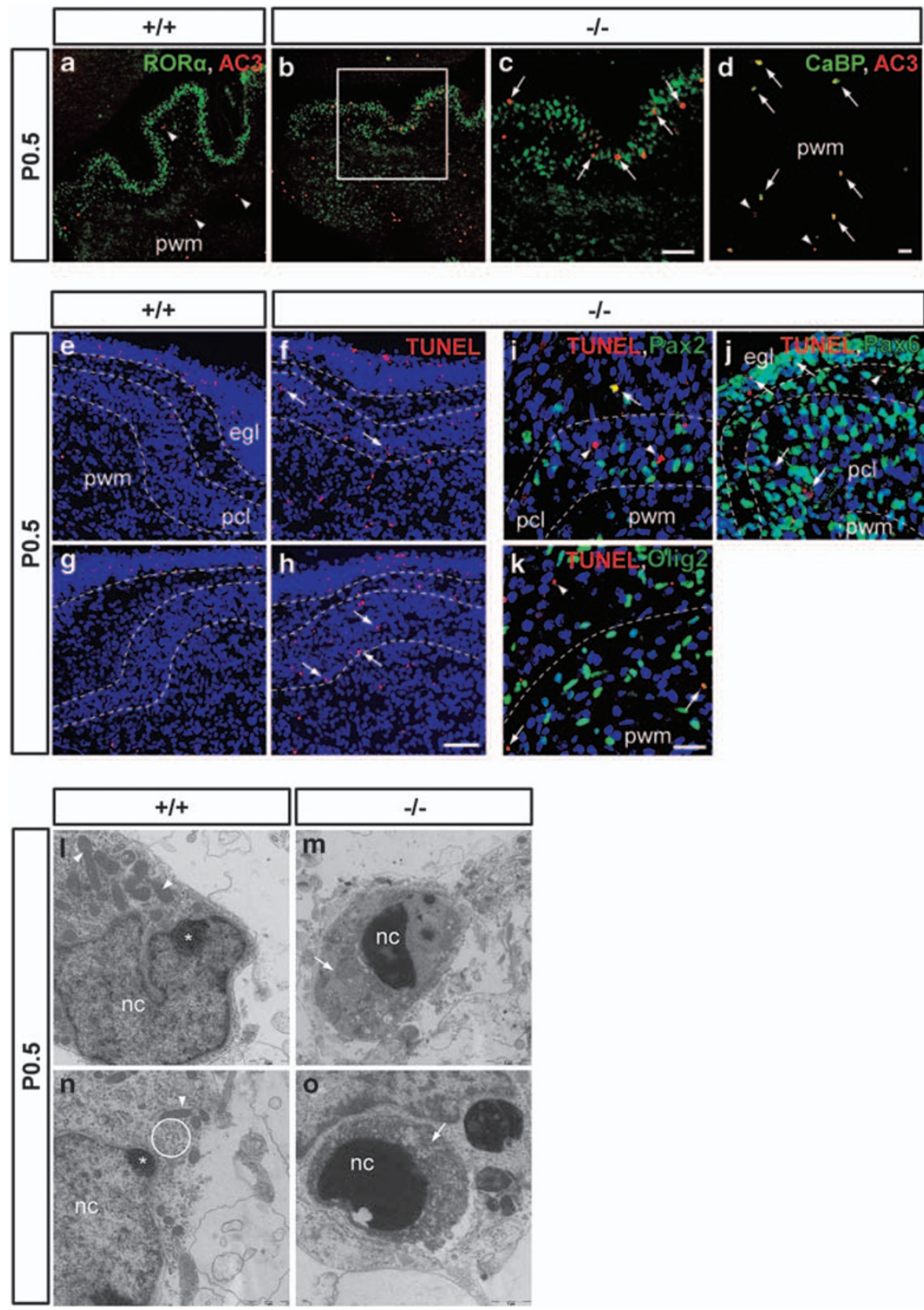

Figure 1 Ebf2 null PCs die by apoptosis shortly after birth. (a-d) Double immunofluorescence on P0.5 sagittal sections, as labeled. In wild-type (wt) mice, cell death, as assessed by activated caspase 3 (AC3) immunoreactivity, is only detectable in the prospective IGL/white matter (pwm), not in the PC layer (arrowheads in a). In the mutant cerebellum, most apoptotic cells are retinoic acid-related orphan receptor $\alpha(\mathrm{ROR} \alpha)$-positive PCs (b, arrows in $\mathbf{c})$. However, dying PCs, positive for calbindin (CaBP), are also found in the mutant pwm (arrows in $\mathbf{d})$. (e-h), TUNEL assay on frontal sections from wt $(\mathbf{e}, \mathbf{g})$ and null mutant $(\mathbf{f}, \mathbf{h})$ cerebella. DNA fragmentation (red fluorescent dots) is evident only in the Ebf2 null PC layer (pcl) (arrows in $\mathbf{f , h}$ ). Note the comparable levels of apoptosis in the external granular layer (egl) of Ebf2 null versus wt control sections. (i-k) In the mutant cerebellum only few TUNEL + cells located in the pwm are positive for Pax2, Pax6 or Olig2 (arrows). (I-o) Electron microscopic imaging of wt (I,n) and Ebf2 null $(\mathbf{m}, \mathbf{o})$ PCs. Wt PCs feature large nuclei with round nucleoli (asterisk). Their cytoplasm is rich in polysomes (circled area) and contains intact mitochondria (arrowheads). Conversely, in the mutant cerebellum $(\mathrm{m}, \mathrm{o})$, numerous apoptotic bodies are found, characterized by a pycnotic nucleus (nc) and condensed cytoplasm (arrow). Scale bar: a-c, $200 \mu \mathrm{m} ; \mathbf{d}, 50 \mu \mathrm{m} ; \mathbf{e}-\mathbf{h}, 100 \mu \mathrm{m} ; \mathbf{i}-\mathbf{k}, 20 \mu \mathrm{m} ; \mathbf{I}, \mathbf{n}, \mathbf{0}, 1 \mu \mathrm{m} ; \mathbf{m}, 2 \mu \mathrm{m}$

RNAi-mediated knock-down of EBF2 using a triple shRNA complementary to the Ebf2 mRNA. The efficiency of this RNAi vector was first validated in Ebf2-overexpressing HEK-293 cells (Figure $4 a$ and b). The experiment was then conducted in neuralized P19 cells that express endogenous Ebf2 (Figure 4c). Our results show that Igf1 transcript levels are decreased in P19 cells after Ebf2 knock-down (Figure 4d).

Similarly, we lipofected RA-treated P19 cells with an Ebf2-expressing vector. After 24 hours, Igf1 expression was analyzed by RT-qPCR. Ebf2-transfected cells showed a fourfold increase in Igf1 transcript levels (Figure 4e), 

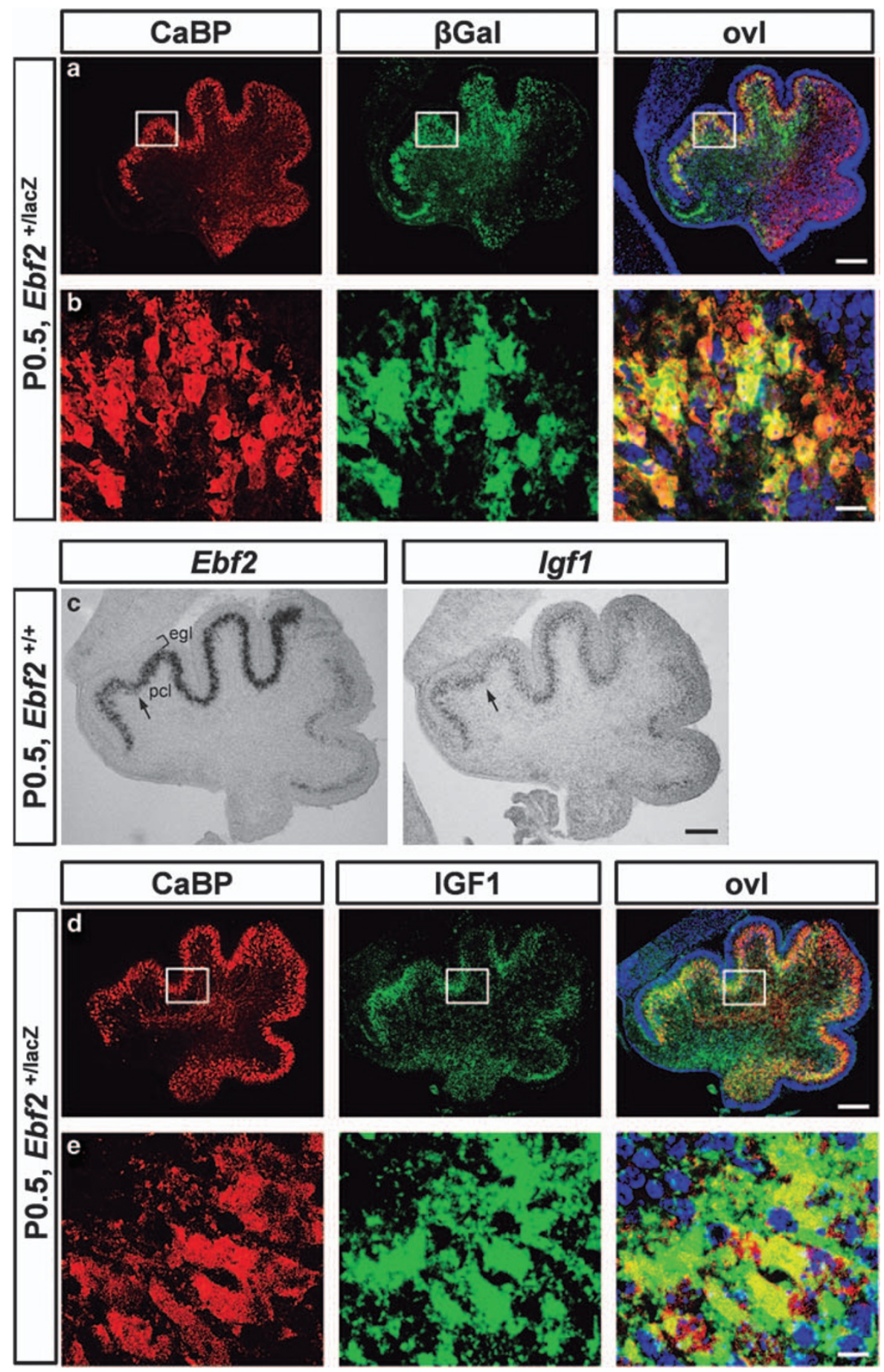

Figure 2 Ebf2 and lgf1 are co-expressed in PCs shortly after birth. (a) $\beta$-gal, a reporter of Ebf2 expression in Ebf2 ${ }^{+/ L a c z}$ heterozygotes, is preferentially expressed in PCs located in the anterior lobe (a, higher magnification in b). (c) In situ hybridization on sagittal cerebellar sections hybridized with an Ebf2 or with an Igf1 cRNA probe. PCs that express Igf1 are also positive for Ebf2 (c). (d) Double immunostaining for CaBP and IGF1 on sagittal cerebellar sections; (e) higher magnification in (d). At both the transcript and protein levels, Ebf2 and IGF1 are found expressed in PCs. pcl: Purkinje cell layer; egl: external granular layer. Scale bar: a,d, $500 \mu \mathrm{m} ; \mathbf{b}, 40 \mu \mathrm{m} ; \mathbf{c}, 200 \mu \mathrm{m} ; \mathbf{e}, 20 \mu \mathrm{m}$

indicating that the effect of EBF2 on Igf1 gene expression is not restricted to PCs and that, in principle, this interaction could be relevant for other Ebf2-expressing neural cell types, both during development and in the adult brain.

EBF2 interacts with the Igf1 gene promoter. Next, we asked if EBF2 regulates Igf1 expression by transcriptional mechanisms. Promoter-reporter gene experiments were performed with COS-7 cells co-transfected with an expression plasmid encoding FLAG-tagged EBF2, and one of three rat Igf1 promoter1-luciferase reporter plasmids containing different $5^{\prime}$ truncations of the promoter. All promoter constructs extended to position +328 , spanning the untranslated region of exon $1^{21}$ (see Figure 5a). 

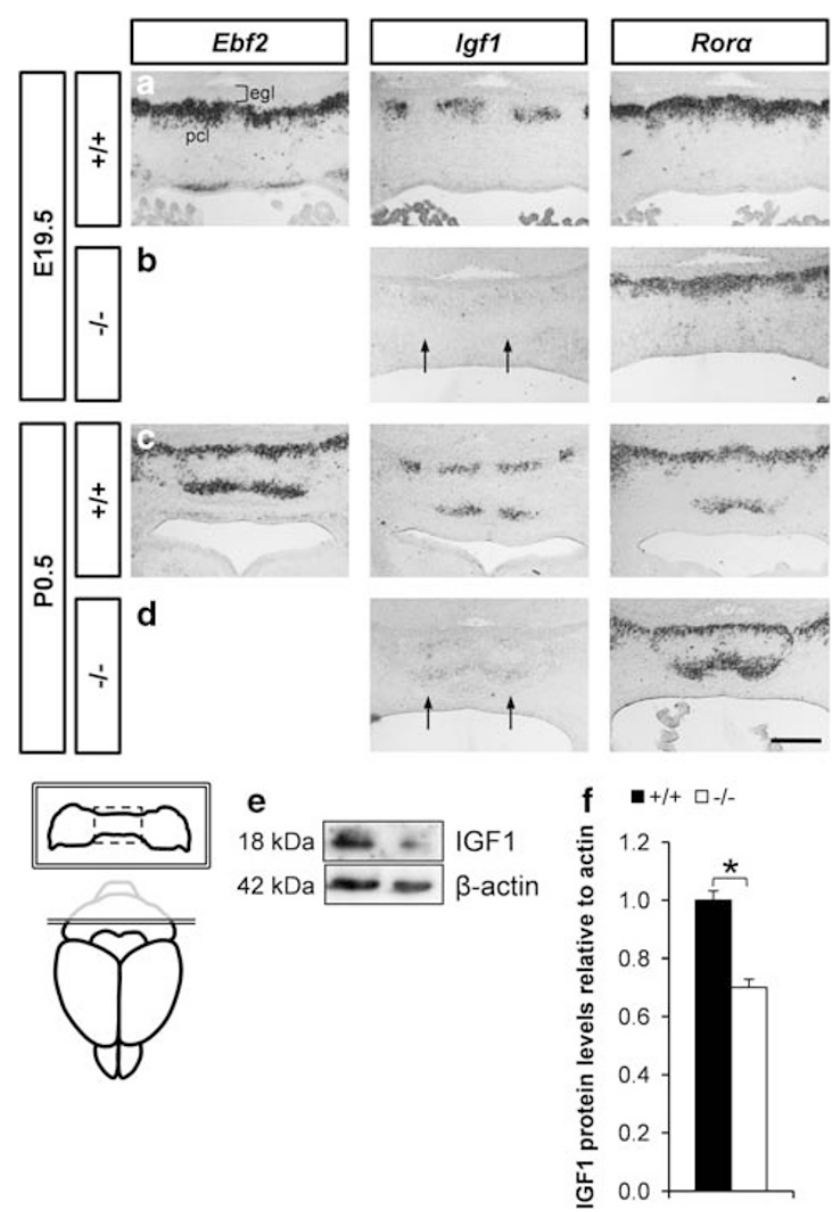

Figure 3 lgf1 expression is downregulated in Ebf2 null PCs. (a-d) In situ hybridization on E19.5 and P0.5 frontal cerebellar sections (sectioning plane sketched at bottom left). In the wt, Igf1 is detectable in a subset of PCs as revealed by comparing its distribution to that of $R O R \alpha$ in adjacent sections. The Igf 1 transcript is almost absent in Ebf2 null PCs (arrows). (e) Western blot analysis of IGF1 protein shows a significant reduction in Ebf2 null lysates compared to the wt (protein levels plotted in f). The experiment was repeated on protein extracts from three wt and mutant cerebella with the S.D. in each case. pcl, Purkinje cell layer; egl, external granular layer. Scale bar: $200 \mu \mathrm{m}$. ${ }^{\star} P<0.05$

The increase in luciferase activity with EBF2 expression was significantly greater with the construct beginning at position -1711 compared with those beginning at positions -823 and -122 (4.5-fold versus 2.6- and 2.0-fold, respectively), consistent with the presence of at least one EBF response element in the -1711 to -823 segment (Figure 5a). As mentioned, two putative target sites, (EBF-A and B) are present at positions -1106 and -1053 relative to the transcriptional start site.

We used the electrophoretic mobility shift assay to determine if EBF2 could bind directly to either EBF-binding site of rat Igf1 promoter 1 . Figure $5 \mathrm{~b}$ shows results obtained using labeled double-stranded oligonucleotides for each DNA sequence, and nuclear protein extracts from COS-7 cells transfected with either FLAG-tagged EBF2 or STAT5b, an established Igf1 transactivator. ${ }^{22}$ As seen in the figure, nuclear proteins containing EBF2 bind to each DNA element, unlike those expressing STAT5b. Competition experiments with unlabeled homologous DNAs showed specificity of binding. Inhibition of binding with a known EBF target site from the OcNC promoter provided further support that EBF2 interacts directly with the two elements from Igf1 promoter 1. In addition, antibody super-shift studies confirmed that EBF2 binds to each oligonucleotide probe.

The Igf1 gene, consisting of 6 exons and 5 introns, is alternatively spliced in different tissues. Different isoforms are generated in the liver (exon 2, liver-secreted isoform) versus other tissues (exon 1, autocrine/paracrine isoform). These isoforms give rise to alternative leader peptides; additional variability is found at the level of exons 4-6, depending on whether or not exon 5 is included in the mature transcript, producing differences in the protein C-terminus. ${ }^{23}$ To determine what splicing isoforms of Igf1 are expressed in the newborn cerebellum, we analyzed wt and Ebf2 null mRNAs. Our results indicate that the prevalent cerebellar isoform contains exon 1 and excludes exons 2 and 5 (Supplementary Figure 4). This isoform is underrepresented in the null cerebellum (Supplementary Figure 4B). Exon 1 of Igf1 is located downstream of the EBF2-binding sites identified in the rat promoter, consistent with the finding that EBF2 drives Igf1 expression in the late embryonic and newborn cerebellum.

PI3K-signaling downregulation in Ebf2 null PCs. The biological functions of IGF1 are mediated through the type I IGF receptor (IGF1R), a receptor tyrosine kinase glycoprotein. Upon IGF1 binding, IGF1R is activated by intermolecular autophosphorylation at four sites within the kinase activation loop, ${ }^{24}$ and phosphorylates the insulin receptor substrates. This, in turn, leads to mitogenactivated protein kinase and phosphatidylinositol 3-kinase (PI3K) activation. ${ }^{25}$ In the latter pathway, Ser473 and Thr308 of protein kinase $B(A K T)$ are sequentially phosphorylated, leading to AKT activation. The PI3K/AKT pathway, is a powerful survival-signaling pathway. ${ }^{26}$ In turn, AKT phosphorylates two pro-apoptotic factors: the BCL2 homolog $B A D$ and the serine kinase GSK3 $\beta$, inactivating them and promoting cell survival. ${ }^{27}$

In the newborn cerebellum, an IGF1/PI3K target, $\mathrm{p}-\mathrm{AKT}^{\text {Ser473 }}$ colocalizes with CaBP expression in PCs, particularly in the anterior lobe (Figure 6a). Unlike IGF1, its predominant subcellular localization is in PC dendrites, as previously described ${ }^{28}$ (Figure $6 b$ ). We examined the effects of Ebf2 nullisomy and Igf1 downregulation on the PI3K pathway in neonatal cerebella. Cerebellar lysates from wt and Ebf2 null mutants were analyzed by western blotting with antibodies specific for AKT-signaling pathway proteins. Our results (Figure $6 \mathrm{c}$ and $\mathrm{d}$ ) indicate that, even though total IGF1R and AKT levels are unchanged in null cerebellar lysates, the amounts of $\mathrm{p}-\mathrm{AKT}^{\mathrm{Thr} 308}$ and $\mathrm{p}-\mathrm{AKT} \mathrm{T}^{\mathrm{Ser} 473}$ are significantly reduced. Similarly, p-GSK3 $\beta^{\text {Ser9 }}$ levels are obviously decreased in the mutant. This result shows that the degree of Igf1 downregulation observed in our mutants is sufficient to inhibit the PI3K/AKT pathway.

IGF1 depletion induces PC apoptosis in wt organotypic tissue culture. Taken together, the above evidence indicates that, in Ebf2 null cerebella, PC death is preceded by IGF1 downregulation. The next question was whether IGF1 is required for PC survival at birth. To address this 
a

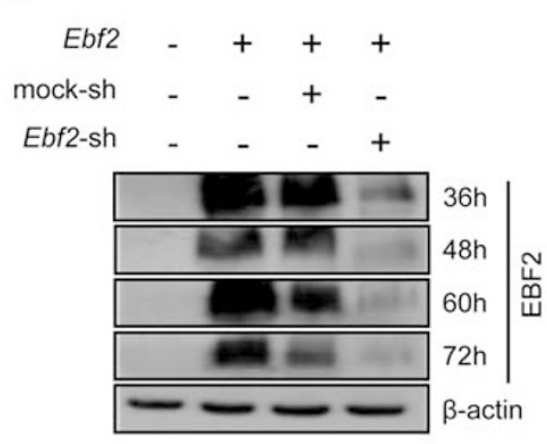

b

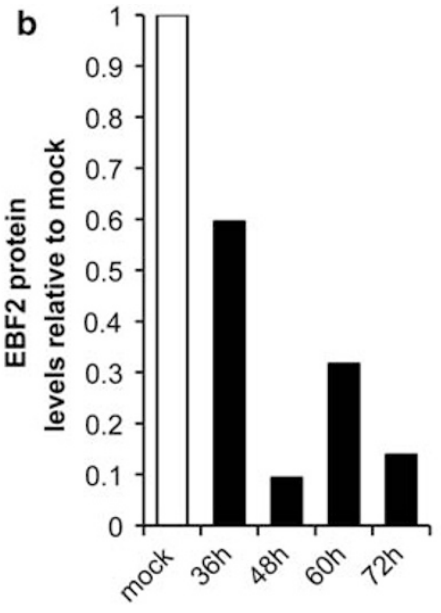

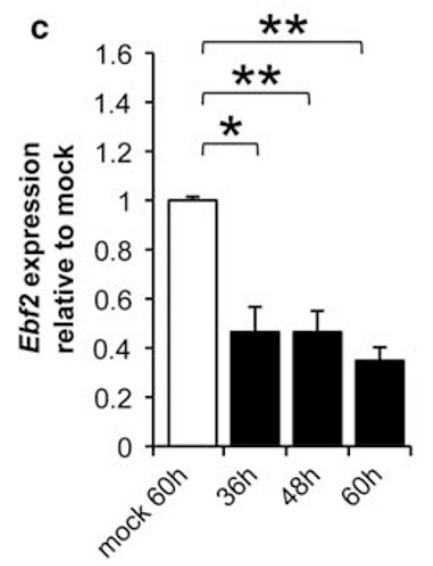
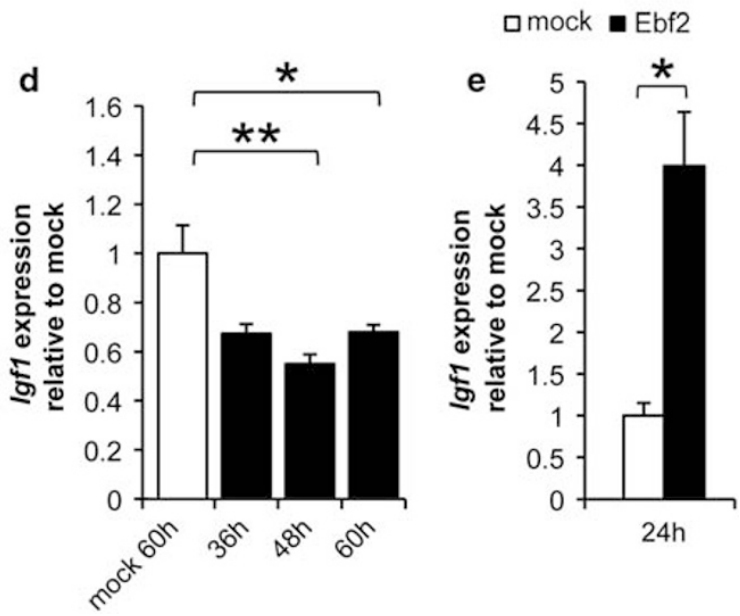

Figure 4 EBF2 activates Igf1 expression in P19 cells. (a) To assess the efficiency of the Ebf2-specific shRNA in downregulating EBF2 protein levels, we overexpressed a flag-tagged Ebf2 in HEK-293 cells that do not exhibit detectable levels of endogenous Ebf2. Western blotting results show that flag-Ebf2-overexpressing HEK-293 cells cotransfected with an Ebf2 shRNA (Ebf2-sh) exhibit a complete downregulation of flag-EBF2 protein levels, compared to cells cotransfected with a mock sh-RNA (mock-sh). (b) Quantitative analysis of the experiment described in (a). Band intensities in sh-treated samples, relative to mock treatment, were measured using the ImageQuant software. All protein levels were also normalized to $\beta$-actin. (c) Time course of endogenous Ebf2 gene expression in neuralized P19 cells after EBF2 knock-down with the Ebf2-sh vector (black columns), relative to the corresponding transcript level in cells treated with mock shRNA (empty column), as measured by RT-qPCR. (d) Time course of endogenous lgf1 gene expression in cells treated as detailed in c (RT-qPCR). (e) Induction of endogenous Igf1 gene expression in neuralized P19 cells transfected with an Ebf2 expression vector after $24 \mathrm{~h}$ (RT-qPCR). (c-e) S.D. are indicated in each histogram. All the experiments shown were repeated three times. ${ }^{*} P<0.05 ;{ }^{* *} P<0.01$

point, we resorted to organotypic cerebellar tissue culture. Sagittal slices were derived from wt E19.5 mouse cerebella and cultured as described in Materials and Methods. Slices from an hemicerebellum were treated with a synthetic IGF1 analog $(\mathrm{H}-1356)$ that acts as a competitive IGF1R inhibitor. ${ }^{29}$ Slices from the contralateral hemicerebellum were treated with vehicle. After 2 days in vitro, cultures were immunostained for $\mathrm{AC} 3$ and $\mathrm{CaBP}$, and analyzed by confocal microscopy. The number of AC3-CaBP double + PCs was scored, revealing a highly significant increase in the number of apoptotic PCs in $\mathrm{H}$-1356-treated sections versus controltreated ones (Figure $7 \mathrm{a}-\mathrm{C}$ ). Our results indicate that IGF1 signal inhibition is sufficient to produce massive PC death in the perinatal cerebellum.

Exogenous IGF1 rescues PC apoptosis in Ebf2 null organotypic slices. Finally, to prove that IGF1 is the pro-survival factor missing in the Ebf2 null cerebellum, we attempted to rescue PC apoptosis in Ebf2 -/- organotypic slices derived as described above. Each hemicerebellum was treated with vehicle or murine-purified IGF1. Again, sections were immunostained for $\mathrm{CaBP}$ and $\mathrm{AC} 3$ and visualized by confocal microscopy. This analysis revealed that the number of apoptotic bodies in the Ebf2 null PC layer was dramatically reduced by IGF1 addition to the culture medium in comparison with vehicle-treated slices (Figure $7 d$ and e, plotted in h). Conversely, IGF1 addition had no significant effect on the baseline levels of PC death scored in wt slices (Figure $7 f$ and $g$, plotted in $\mathrm{h}$ ).

\section{Discussion}

This study confirms that EBF2 controls neonatal PC survival in vivo and in organotypic slices by inhibiting apoptosis, and 


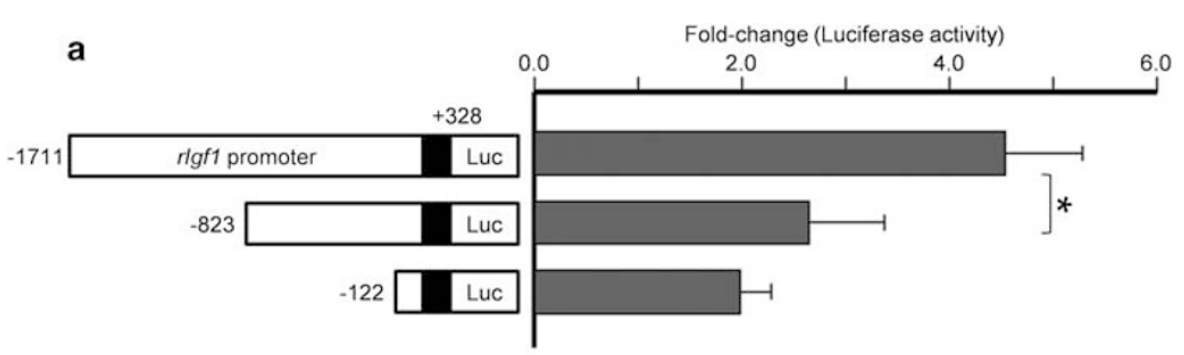

b
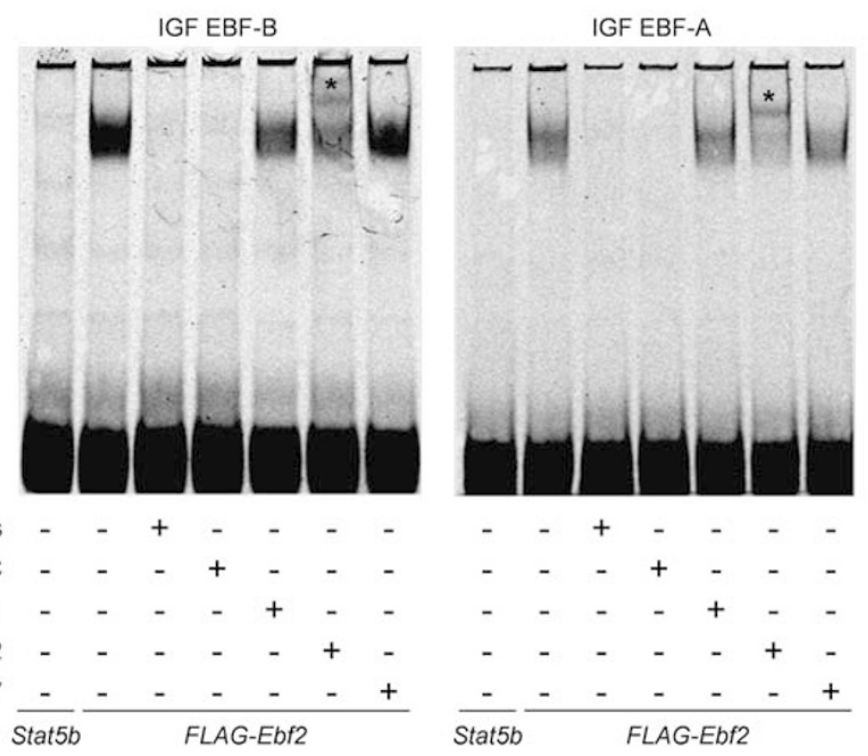

Figure 5 EBF2 binds two sequences of Igf1 promoter 1 and enhances promoter activity. (a) Results of luciferase assays in COS-7 cells transiently transfected with an expression plasmid for Ebf2 (or an empty vector) and with the Igf1 promoter-reporter plasmids, as sketched. The graph summarizes results of four independent experiments (mean \pm S.E.), each performed in duplicate and normalized for total protein content with the S.D. in each case. The asterisks indicate significant difference in EBF2-induced promoter activity $(P<0.03)$ in comparison to the -1711 promoter-reporter plasmid that spans putative-binding sites IGF EBF-A and -B. Basal luciferase values ranged from 10000 to 135000 relative light units/10 s. (b) Results of gel-mobility shift assays using IR-labeled double-stranded oligonucleotides for either IGF EBF-A or -B of rat Igf1 promoter 1. The DS oligos were incubated with nuclear protein extracts from COS-7 cells transfected with expression plasmids encoding FLAG-tagged EBF2 or the unrelated STAT5b. Protein-DNA complexes are observed only with nuclear extracts from EBF2-expressing cells. 50-fold excess of unlabeled homologous oligonucleotide and a previously described EBF-binding site of the OcNC gene effectively compete off binding, whereas an Oct-1 recognition site does not. A supershift of each protein-DNA complex $\left(^{*}\right)$ is seen with an antibody to the FLAG-epitope, but not with an irrelevant antibody directed against 11 amino acids from the T7 phage gene 10 leader peptide. Unbound probe is observed at the bottom of each gel

indicates that EBF2 activates Igf1 expression in various cell lines, interacting with a distal Igf1 promoter element. In late embryogenesis and at birth, Ebf2 and Igf1 colocalize in the PC layer, particularly in the anterior and intermediate lobes (Figure 2). Wt organotypic cultures plated just before birth are strictly dependent on IGF1 for PC survival. Death in Ebf2 null slices is rescued by adding purified IGF1 to the medium. Taken together, these results indicate that the increase in PC apoptosis observed in Ebf2 null mice stems from Igf1 downregulation in mutant PCs, and strongly suggest that EBF2 supports PC survival by directly promoting Igf1 expression in these cells. Although EBF2 is by no means the only TF-regulating Igf1 expression in the cerebellum, ${ }^{30,31}$ it appears to act as a critical regulator of Igf1 transcription and PC survival at birth.

Why the first postnatal day? Neonatal PCs are under a great deal of metabolic stress. The massive presence of ribosomes in the cytoplasm of newborn PCs has been firmly established. At this stage, PCs start to develop their massive dendritic tree, ${ }^{32}$ elaborate existing synaptic contacts with olivocerebellar fibers and begin to establish their first efferent synaptic contacts with deep nuclear neurons. ${ }^{33}$ Experiments conducted on cerebellar organotypic slices have revealed that these cells go through a critical period between $\mathrm{P} 1$ and $\mathrm{P} 7^{34}$ and may require trophic support from IGF1 to sustain this transition.

Why the anterior lobe? PC death at birth is mostly clustered in the anterior lobe. Although all PCs share common morphological features, they are in fact subdivided into molecularly defined sub-classes arranged into alternate zones and stripes. ${ }^{35}$ Zebrin II is the best characterized marker of PC subsets. Zebrin II + and - PCs feature different properties with respect to cell survival, both under physiological and pathological conditions. ${ }^{36}$ Ebf2, a marker of Zebrin II- $\mathrm{PCs}^{12}$ is expressed in an anterior-to-posterior gradient at birth, colocalizing with $\lg f 1$, and may be selectively required for the survival of zebrin II- PCs. 


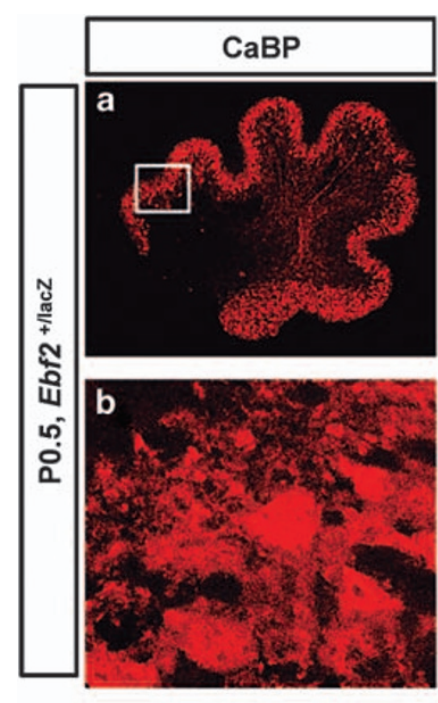

C

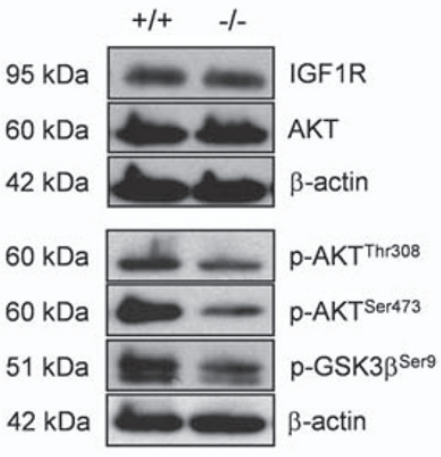

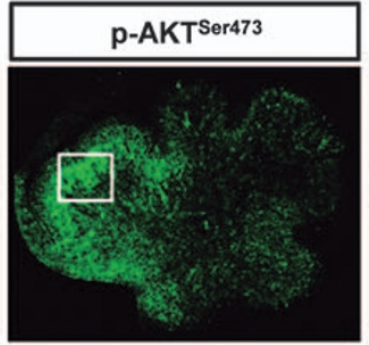
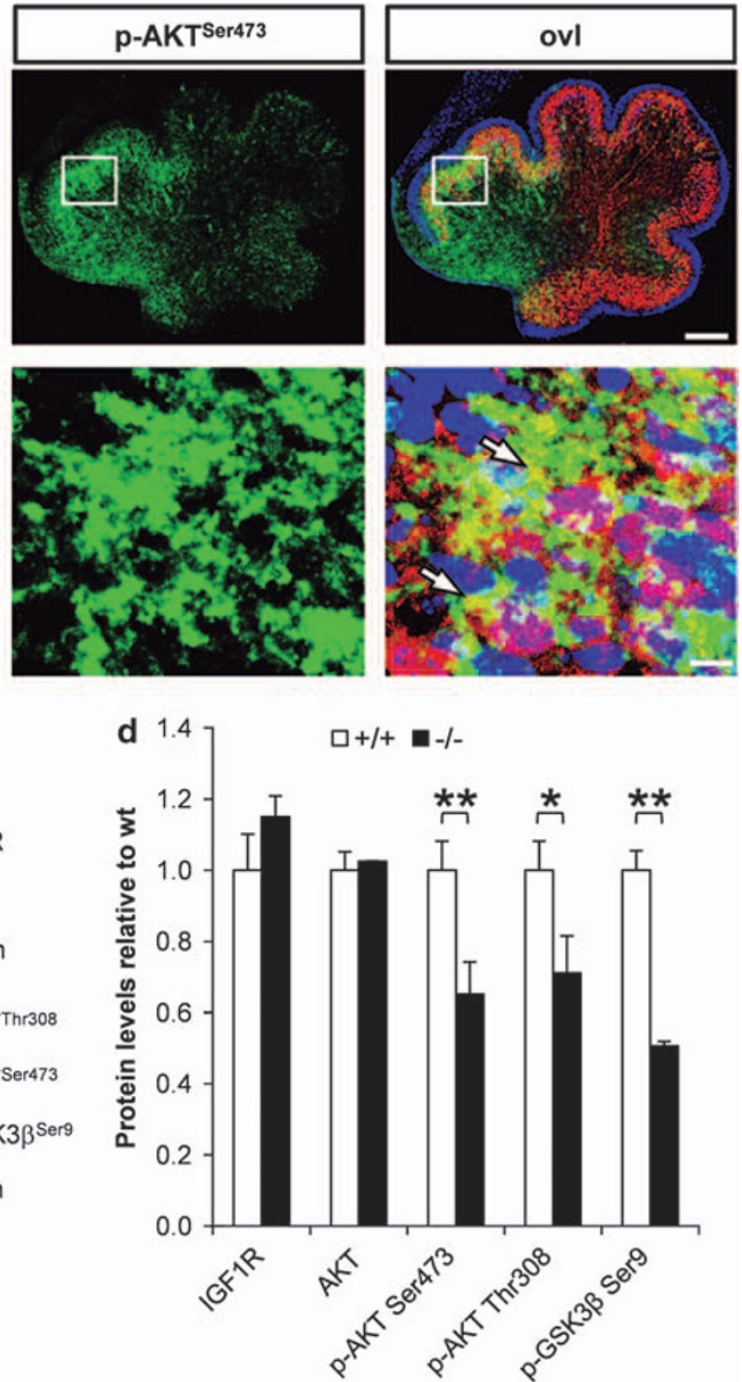

Figure 6 Reduced AKT1 phosphorylation in the Ebf2 null cerebellum. (a) P0.5 sagittal sections from an Ebf2 ${ }^{+/ L a c Z}$ cerebellum immunostained for CaBP and the IGF1 effector AKT, phosphorylated in Ser ${ }^{473}$. Phospho-AKT staining in PCs reveal the activation of the IGF1R/PI3K-signaling pathway in these cells, specifically in dendrites (arrows in b). (c) Cerebellar protein extracts from wt and Ebf2 null mice were analyzed by western blotting at the same stage (P0.5). The levels of AKT phosphorylation at both Ser473 and Thr308, and GSK3 $\beta$ phosphorylation at Ser9 are dramatically reduced in Ebf2 -l- lysates, whereas total AKT and IGF1R levels are unchanged. (d) The intensity of immunoreactivity was quantified using the ImageJ software. Protein levels were normalized to $\beta$-actin. Each immunoblotting experiment was repeated on protein extracts from four independent wt and null mutant mice. S.D. is shown in each case. Size bars: (a) $500 \mu \mathrm{m}$; (b) $20 \mu \mathrm{m}$. ${ }^{\star} P<0.05$; ${ }^{* \star} P<0.01$

Does IGF1 affect PC survival cell autonomously? Besides PCs (present paper and 12), other apoptotic cells are found in the prospective IGL/WM of newborn Ebf2 mutants. At birth, this layer contains a small quota of postmigratory granule cells, numerous unipolar brush cells, many GABA interneurons and their progenitors, and oligodendrocyte precursors. Dying cells belong to each of these classes, with a prevalence of PCs that are completing their developmental migration. Thus, while a non cell-autonomous effect on PC survival cannot be excluded, the most parsimonious explanation is that PCs die due to an autocrine/paracrine deficit in Igf1 expression, likely increasing their susceptibility to metabolic stress during the first hours of postnatal life.

The importance of a local source of IGF1. The lack of a significant correlation between serum and cerebrospinal fluid
IGF concentrations in normal people and patients with pituitary disorders suggests that endocrine IGF ligands might not contribute crucially to IGF signaling in the CNS. ${ }^{37}$ This underlines the importance of local sources of IGF1 in the nervous system. Although long-term administration of supraphysiological amounts of IGF1 can protect PCs in an ataxia model, ${ }^{31}$ our data suggest that, physiologically, local Igf1 transcription is key to neuronal survival at defined stages of development. Our findings indicate that PC survival in the Ebf2 null mouse depends on the local activation of Igf1 transcription at birth.

Besides the cerebellar defects described here, Ebf2 null mutants feature an impairment in gonadotropic axis and peripheral nerve development. ${ }^{10}$ IGF1 has been implicated in gonadotropic-releasing hormone $(\mathrm{GnRH})$ neuron development and may control $\mathrm{GnRH}$ transcription and release. ${ }^{38}$ 

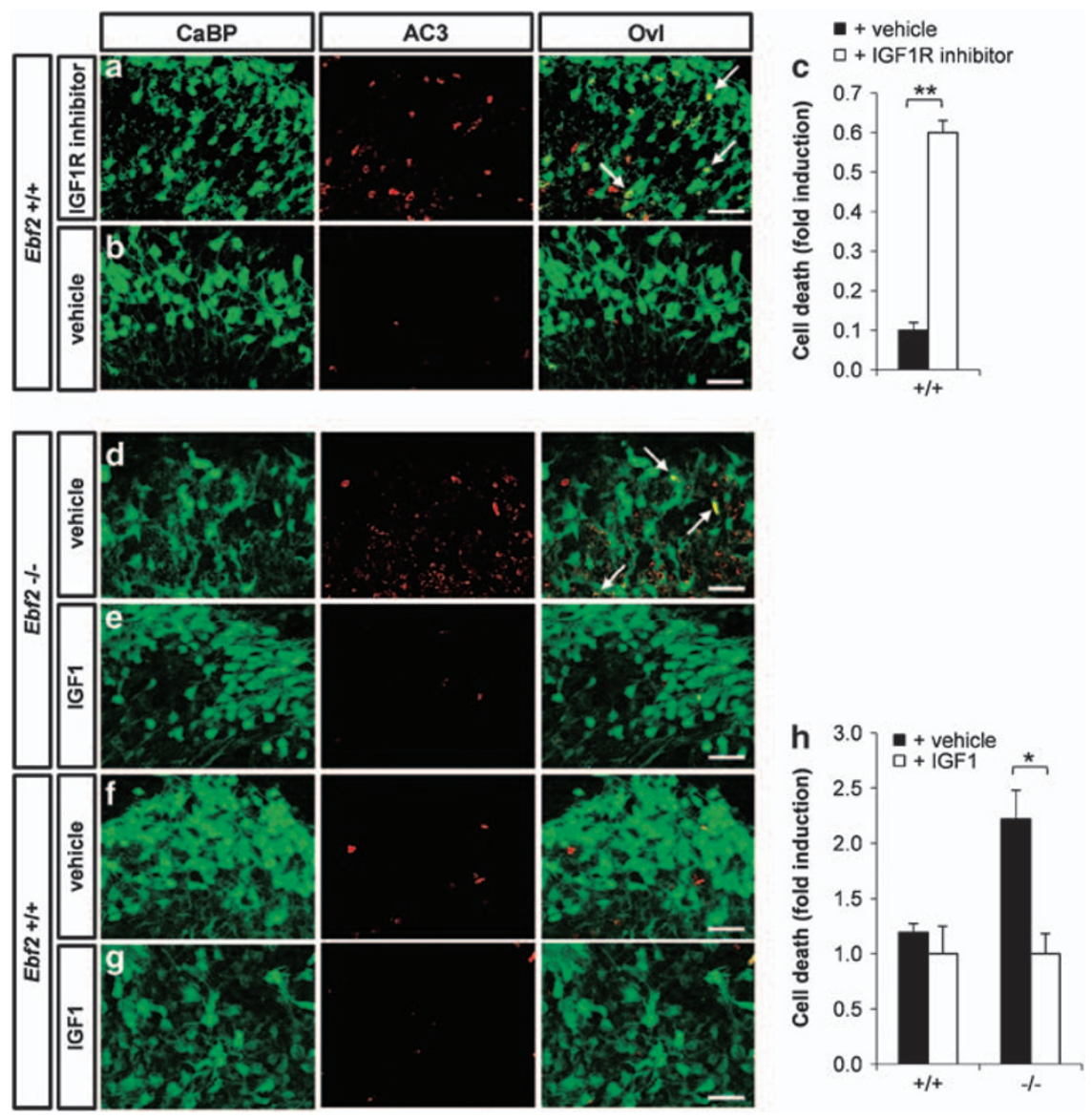

Figure 7 IGF1 is essential for PC survival in organotypic cultures of the perinatal cerebellum. (a,b) E19.5 wt cerebella were divided in two parts and each hemicerebellum was treated with an IGF1 competitor (H1356 Bachem) (a) or vehicle $(1 \times \mathrm{PBS})(\mathbf{b})$, and maintained in culture for 2 days. The number of apoptotic cells is revealed by immunostaining for $\mathrm{CaBP}$ and active caspase 3 (AC3). Cell death is sharply increased in competitor-treated slices (plotted in c). (d-g) Cerebella from Ebf2 null mutants and wt controls were divided in two parts and each hemicerebellum was treated with vehicle $(1 \times$ PBS in d,f) or murine purified IGF1 (e,g), and maintained in culture for 2 days. The number of apoptotic PCs was dramatically reduced in IGF1-treated Ebf2 null slices (e) in comparison to vehicle-treated null slices (d). Conversely, IGF1 treatment had no effect on cell death in control slices when compared to vehicle treatment (f and $\mathbf{g}$, respectively). (c,h) Statistical significance of both experiments was estimated computing the ratio of AC3-positive cells located in the PC layer to the total number of CaBP-positive PCs, relative to IGF1 treatment. All treatments were repeated three times for each genotype. The S.D. is indicated in all cases examined. ${ }^{*} P<0.05,{ }^{\star \star} P<0.01$. Size bar: $50 \mu \mathrm{m}$

Moreover, the Ebf2 null peripheral nerve is defective in axon fasciculation and myelination. ${ }^{10}$ Published studies implicate IGF1 in Schwann cell differentiation. ${ }^{39}$ Further studies will be required to establish whether EBF2 controls GnRH neuron and peripheral nerve development by promoting lgf1 expression.

The controversial roles of IGF1 in neuronal survival and degeneration. While our results indicate that IGF1 is acutely required to support PC survival at birth, the role of IGF1 with respect to neuronal survival and aging is debated. Genetic evidence indicates that a reduction in insulin/IGF1 signaling (IIS) increases longevity and delays the onset of protein-aggregation-mediated toxicity in invertebrates and mammals alike. IGF1R mutation correlates with lifespan extension in the nematode and fruitfly. Similarly, heterozygous IGF1R mutant mice live significantly longer than controls (reviewed by Cohen and Dillin ${ }^{40}$ ). This paradox suggests that each organism has an optimal level of IIS that maximizes its reproduction, fitness and longevity, and that IIS levels either higher or lower than the optimal rate interfere with metabolism, causing disease and shortening lifespan.

Taken together, these observations emphasize the importance of achieving a fine regulation of Igf1 gene expression. Transcriptional control by EBF2 is a novel player in the homeostatic regulation of IIS. EBF2 and other EBF TFs, that are expressed at many sites in development and after birth, ${ }^{18}$ may participate in lgf1 regulation at different stages and locations, in physiology and disease.

\section{Materials and Methods}

Animal care. All experiments described in this paper were conducted in agreement with the stipulations of the San Raffaele Scientific Institute Animal Care and Use Committee.

Mouse genetics. All experiments were carried out on F1 hybrids obtained by crossing Ebf2 $+/$ - pure-bred FVB/N (N9) females with Ebf2 $+/$ - pure-bred C57BL/6J males. ${ }^{10}$ All studies were conducted using coisogenic control littermates. 
This hybrid strain was chosen to obviate the low fertility and poor maternal behavior of C57BL/6J heterozygous mothers.

Tissue preparation. Newborn mice were anesthetized with Avertin (Sigma, St Louis, MO, USA) and perfused with $0.9 \% \mathrm{NaCl}$ followed by $4 \%$ paraformaldehyde (PFA). Embryos were fixed overnight by immersion in 4\% PFA. Fixed tissues were rinsed three times in $1 \times$ PBS, cryoprotected in $30 \%$ sucrose overnight, embedded in OCT (Bioptica, Milan, Italy), and stored at $-80^{\circ} \mathrm{C}$, before sectioning on a cryotome $(20 \mu \mathrm{m})$

Electron microscopy. The samples from saline-perfused pups were fixed for $2 \mathrm{~h}$ at $4{ }^{\circ} \mathrm{C}$ with $4 \%$ paraformaldehyde and $2.5 \%$ glutaraldehyde in $125 \mathrm{mM}$ cacodylate buffer, then post fixed $(1 \mathrm{~h})$ with $2 \% \mathrm{OsO}_{4}$ in $125 \mathrm{mM}$ cacodylate buffer Samples were washed and embedded in Epon. Electron microscopy analysis was performed on ultrathin transverse sections made on a Leica Ultracut UCT ultramicrotome, stained with uranyl acetate and lead citrate. Slides were examined in a Leo912 electron microscope.

Organotypic cultures. F1 fetuses from embryonic day 19 were used. Fetuses were obtained by cesarean section from pregnant mice anesthetized with Avertin (Sigma). After killing the mice, brains were dissected out into cold Dulbecco's modified Eagle's medium (DMEM), and meninges were removed. Cerebellar parasagittal slices (300- $\mu \mathrm{m}$ thick) were cut on a Mcllwain tissue chopper and separated gently into cold PBS. The slices were cultured on the membrane of a $30 \mathrm{~mm}$ Millipore culture insert (Millicell, Millipore, Billerica, MA, USA; pore size $0.4 \mu \mathrm{m}$ ) in $6 \mathrm{~cm}$ culture dishes containing $1 \mathrm{ml}$ of medium composed of $50 \%$ basal medium with Earle's salts (Sigma), 25\% HBSS (Sigma), 25\% horse serum (Euroclone, Milan, Italy), L-glutamine (1 $\mathrm{mM}$, Euroclone), and $5 \mathrm{mg} / \mathrm{ml}$ glucose (Sigma) at $37^{\circ} \mathrm{C}$ in an atmosphere of humidified $5 \% \mathrm{CO}_{2}$. Tissue slices were left in culture for 2 days before further manipulations and treated immediately in the medium with murine-purified IGF1 (100 ng/ml, Peprotech, Rocky Hill, NJ, USA), IGF1 peptide competitor (30 $\mu \mathrm{M}$, CYAAPLKPAKSC), as previously described, ${ }^{29}$ or vehicle (1X PBS).

Immunohistochemistry and TUNEL staining. For dual immunofluorescence, floating cryosections were rinsed three times in $1 \times$ PBS, preincubated in $15 \%$ fetal bovine serum (FBS), $0.2 \%$ Triton X-100, $1 \times$ PBS and incubated overnight at $4^{\circ} \mathrm{C}$ with the two primary antibodies. Sections were then washed $6 \times 10 \mathrm{~min}$ in $1 \times$ PBS and incubated for $2 \mathrm{~h}$ at room temperature with the two secondary antibodies (Alexa 546 anti-rabbit lgG, 1:1000, and Alexa 488 antigoat IgG, 1:1000). Cerebellar organotypic slices were fixed in $4 \%$ PFA for $1 \mathrm{~h}$ at room temperature. After $1 \times$ PBS washing, the slices were taken off the Millicell and processed for dual immunofluorescence. The slices were preincubated in $15 \%$ goat serum, $0.2 \%$ Triton X-100, $1 \times$ PBS and incubated overnight at $4{ }^{\circ} \mathrm{C}$ with the first antibody (rabbit anti-active Caspase 3). Sections were then washed $6 \times 10 \mathrm{~min}$ in $1 \times$ PBS and incubated for $2 \mathrm{~h}$ at room temperature with the secondary antibody (Alexa 546 anti-rabbit IgG, 1:1000), the slices were washed $6 \times 10$ min in $1 \times$ PBS and incubated with the second primary antibody (rabbit anti-calbindin) that was labeled before by using a Zenon Alexa Fluor 488 goat anti-rabbit IgG1 kit (Invitrogen, Carlsbad, CA, USA) and counterstained with Hoechst (Sigma). In controls either primary antibody was replaced with normal serum. The slices were mounted in Mowiol (Calbiochem, La Jolla, CA, USA) and analyzed using a Leica confocal microscope. TUNEL assay was performed using ApopTag kit (Millipore) according to the manufacturer's instructions. Primary antibodies were as follows: rabbit anti-active Caspase 3, 1:300 (BD Pharmingen, San Jose, CA, USA); goat anti-Ror $\alpha, 1: 100$ (Santa Cruz Biotechnology, Santa Cruz, CA, USA); rabbit antiCalbindin, 1:1000 (Swant, Bellinzona, Switzerland); rabbit anti- $\beta$-galactosidase, 1:10000 (Cappel, Costa Mesa, CA, USA); goat anti-IGF1, 1:100 (R\&D Systems, Minneapolis, MN, USA); rabbit anti-p-AKT ${ }^{\text {Ser473 }} 1: 50$ (Cell Signaling Technology, Danvers, MA, USA). The signal from anti- $\beta$-galactosidase, anti-IGF1 and anti-p$\mathrm{AKT}^{\mathrm{Ser} 473}$ primary antibodies was amplified using the Tyramide Signal Amplification Kit (Perkin Elmer, Waltham, MS, USA).

Cell counts on cryotome sections. The quantitation of TUNEL- and AC3positive cells (Figure 1) was obtained counting complete series of sections from wt and mutant PO cerebella $(n=3)$. Statistical analysis of mean cell numbers and S.D. in wt versus null mutant sections was conducted using a two-tailed $t$-test, equal variance. On average, TUNEL staining revealed $\sim 15 \%$ more unequivocally positive cells than AC3 immunostaining in both wild type and mutant cerebella.
In situ hybridization. Digoxygenin-labeled riboprobes were transcribed from plasmids containing Ebf2, Igf1 and Rora (a gift of B A Hamilton) cDNAs. In situ hybridizations were performed as described by Pringle and Richardson (http:// www.ucl.ac.uk/ ucbzwdr/Richardson.htm). Briefly, the sections were permeabilized with proteinase $\mathrm{K}(0.5 \mu \mathrm{g} / \mathrm{ml}, 7 \mathrm{~min})$, post-fixed with $4 \%$ paraformaldehyde, rinsed three times in $1 \times$ PBS, and hybridized with riboprobes in $50 \%$ formamide, $3 \times$ SSC, $10 \%$ dextran sulfate, and $500 \mu \mathrm{g} / \mathrm{ml}$ tRNA solution at $56^{\circ} \mathrm{C}$ overnight. The sections were washed twice in $50 \%$ formamide/ $2 \times \mathrm{SSC}$, treated with Ribonuclease A (20 $\mu \mathrm{g} / \mathrm{ml}, 30 \mathrm{~min})$, washed in $50 \%$ formamide $1 \times$ SSC, rinsed in $2 \times \mathrm{SSC}$, and incubated with an antidigoxigenin alkaline phosphataseconjugated antibody at $4^{\circ} \mathrm{C}$ overnight. After incubation, the sections were washed three times in $100 \mathrm{mM}$ Tris- $\mathrm{HCl}(\mathrm{pH} 7.5), 150 \mathrm{mM} \mathrm{NaCl}$, then once in $100 \mathrm{mM} \mathrm{NaCl}$, $100 \mathrm{mM}$ Tris- $\mathrm{HCl}, \mathrm{pH} 9.5,50 \mathrm{mM} \mathrm{MgCl}_{2}$. For the color development reaction, 4-nitroblue tetrazolium chloride and 5-bromo-4-chloro-3-indolyl phosphate were used as substrates.

Cell culture and DNA transfection. The P19 cell line was maintained in MEM- $\alpha$ (Invitrogen) supplemented with 10\% FBS (Invitrogen). Hek293 and COS-7 cell lines were maintained in DMEM supplemented with $10 \%$ fetal FBS (EuroClone). P19 and Hek293 cells were transfected with Lipofectamine2000 (Invitrogen), and COS-7 with Transit-LT1 (Mirus, Madison, WI, USA) according to manufacturers' recommendations. Depending on the experiment, $\mathrm{P} 19$ cells were grown in $5 \% \mathrm{FBS}$ and $10^{-6} \mathrm{M}$ all-trans retinoic acid (RA, Sigma) for $24,36,48,60 \mathrm{~h}$ after transfection and sorted for GFP to study shRNA-enriched population. pcDNA3-Flag-Ebf2 construct was a gift from Matthias Kieslinger (University of Munich). pcDNA3 vector, used as a control, was from Clontech (Mountain View, CA, USA).

RNA interference. Single-stranded DNA oligos encoding the pre-miRNAs were annealed according to the manufacturer's instructions (Invitrogen). Pre-miRNA double-stranded oligos were ligated into pcDNA 6.2-GW/-EmGFP-miR vector (Invitrogen), that contains an EGFP gene. A Pre-miRNA containing a non-targeting sequence (Invitrogen) was used as a negative control. These miRNA vectors were a gift from Matthias Kiesslinger. miRNA targets against Ebf2 were designed using the BLOCK-iT RNAi designer. Three oligos matching three different sites of the Ebf2 mRNA were selected to maximise knockdown efficiency. This triple miRNA may cross-react with two other members of the Ebf gene family. The three miRNAs were cloned sequentially in the $3^{\prime} U T R$ of the GFP gene, according to the supplier's instructions. The triple shRNA was transfected via Lipofectamine2000 (Invitrogen) according to the manufacturer's recommendations. Transfected P19 cells were neuralized with RA (Sigma), then sorted for GFP expression at different time-points and lysed for RNA extraction with RNeasy MiniKit Plus (Qiagen, Valencia, CA, USA), according to the manufacturer's instructions.

Reverse transcription (RT)-PCR assay. Total RNA was extracted with RNeasy MiniKit Plus (Qiagen), according to the manufacturer's instructions. $1-1.5 \mu \mathrm{g}$ of total RNA was retro-transcribed using first strand CDNA MMLVRetrotranscriptase (Invitrogen) and random primers. Each CDNA was diluted $1: 10$, and $3 \mu$ l were used for each reaction. mRNA quantization was performed using the LightCycler480 SYBR Green I Master Mix (Roche, Mannheim, Germany) on a LighCycler480 instrument (Roche). The following oligonucleotide primers were used: Ebf2 TaqMan probe (Applied Biosystem, Foster City, CA, USA), Igf1 (Igf1-F: 5'-GGAGACTGGAGATGTACTGTG; Igf1-R: 5'-GTACTTCCTTCTGAGTC TTGG-3') and Gapdh (Gapdh-F: 5'-TGAAGCAGGCATCTGAGGG; Gapdh-R: $5^{\prime}$ - CGAAGGTGGAAGAGTGGGAG-3'). Each gene was analyzed in triplicate, and each experiment was repeated at least three times. Data analysis was performed with the $\Delta \Delta C_{\mathrm{t}}$ method. All RNA levels were normalized based upon GAPDH transcript levels. For semi-quantitative RT-PCR the following oligonucleotide primers were used: Igf1-Ex1-F: 5'-TCTGCCTCTGTGACTTCTTG-3'; Igf1-Ex2-F: 5'-TCTGACCTGCTGTGTAAACG-3'; Igf1-Ex4-F: 5'-GTTGCTTCCGGAGCTGTGAT-3'; Igf1-Ex4-R: 5'-ATCACAGCTCCGGAAGCAAC-3'; Igf1-Ex6-R: 5'-GGTAGGTGTTTCGA TGTाTG-3'; Hprt-F: 5' - TCCCTGGTTAAGCAGTACAG-3'; Hprt-R: 5'-GACGCAGCAA CTGACATTTC-3'.

Electrophoretic mobility shift assays. Electrophoretic mobility shift assays were performed as described ${ }^{22}$ with $2.5 \mu \mathrm{g}$ of COS-7 nuclear proteins prepared from cells transfected with an expression plasmid for FLAG-epitope tagged EBF2 or for FLAG-Stat5b (negative control), and 5'-labeled infrared (IR) double-stranded oligonucleotide probes from putative EBF-binding sites in rat IGF1 gene promoter 1, IGF EBF-A from -1110 to -1087 with respect to the most 
$5^{\prime}$ transcription start site: ${ }^{21}$ top strand, $5^{\prime}$-TCAAATCCCCCGGGAGTGGAGAGT-3', and IGF EBF-B from -1058 to -1037: top strand, $5^{\prime}$-TCCATCTCCCCTGGGAA AGCACT $-3^{\prime}$ with consensus sites underlined. After incubation of proteins and DNA for $60 \mathrm{~min}$ at $4{ }^{\circ} \mathrm{C}$, products were separated by electrophoresis through nondenaturing, $5 \%$ polyacrylamide gels in $0.5 \mathrm{XTBE}$ ( $45 \mathrm{mM}$ Tris, $45 \mathrm{mM}$ boric acid, and $1 \mathrm{mM}$ EDTA, pH 8.3) at $130 \mathrm{~V}$ for $0.5 \mathrm{~h}$ at $20^{\circ} \mathrm{C}$. Results were detected using an infrared imager (LiCoR Odyssey), and v3.0 analysis software (LiCoR Biosciences, Lincoln, NB, USA). Antibody super-shift and competition experiments were performed as described. ${ }^{22}$ The top strand of each heterologous competitor oligonucleotide is as follows: EBF target, OcNC: $5^{\prime}$-AAGGGAGAGTCCCTAGG GAGCTTGGGAGGGGCCA-3', Oct-1: 5'-TTTTAGAGGATCCATGCAAATGGACG TACG-3' . Antibodies ( $1 \mu \mathrm{g}$ added per lane) were directed against FLAG M2 (Sigma) and T7 epitopes (Novagen, Darmstadt, Germany).

Luciferase assays. COS-7 cells were co-transfected with an expression plasmid for EBF2 or empty vector (pcDNA3), and with one of three promoterreporter plasmids encoding different segments of rat IGF1 promoter 1 and the entire non-coding region of exon 1, as depicted in Figure 5 . After incubation for $40 \mathrm{~h}$, cells were harvested and lysates used for luciferase assays. All results were normalized for total cellular protein values measured by BCA assay (Pierce, Rockford, IL, USA).

Western blotting. HEK293 cells were harvested 36, 48, 60 and $72 \mathrm{~h}$ after transfection, and the collected cells were frozen at $-80^{\circ} \mathrm{C}$. For western Blot experiments, cells or neonatal cerebellar were lysed in $300 \mu$ l of RIPA buffer containing protease inhibitors. Samples were sonicated and centrifuged at 13000 r.p.m. for $15 \mathrm{~min}$ at $4^{\circ} \mathrm{C}$ and supernatants were collected. Protein concentration was determined by the BCA assay (Pierce). Lysates were mixed with sample buffer (125 mM Tris-HCl pH 6.8; 0.1 M 2-mercaptoethanol; 2\% SDS; 20\% glycerol; $25 \mathrm{mg} / \mathrm{ml}$ Bromphenol Blue), boiled for $15 \mathrm{~min}$ and separated on an SDS-polyacrylamide gel at $10 \%$. Proteins were transferred on a PVDF membrane (Millipore). Western blotting were performed with the following antibodies: goat anti-IGF1, 1:100 (R\&D Systems), rabbit anti-EBFs, 1:1000 (Eurogentec, Liege, Belgium), rabbit anti-IGF1R, 1:1000 (Cell Signaling Technology), rabbit anti-AKT, 1:1000 (Cell Signaling Technology), rabbit anti-p-AKT Thr308, 1:500 (Cell Signaling), rabbit anti-p-AKT ${ }^{\text {Ser473 }}, 1: 500$ (Cell Signaling Technology), rabbit anti-p-GSK3 $\beta^{\text {Serg }}, 1: 500$ (Cell Signaling Technology), mouse anti- $\beta$-actin, 1:4000 (Sigma). The secondary antibodies used were a goat anti-rabbit HRP-conjugated antibody, 1:30 000 (Bio-Rad Laboratories, Hercules, CA, USA) and a sheep anti-mouse HRP-conjugated antibody, 1:3000 (Santa Cruz Biotechnology) and a sheep anti-goat HRP-conjugated antibody, 1:2000 (DAKO, Glostrup, Denmark). Blots were developed with the LiteAblot substrate (EuroClone).

Statistical analysis. Statistical significance was determined by the Student's $t$-test and Pearson's $\chi^{2}$-test, for the analysis of organotypic cultures, with a threshold for significance set to $P \leqslant 0.05$. All data are plotted as the mean \pm S.D., unless stated otherwise.

\section{Conflict of interest}

The authors declare no conflict of interest.

Acknowledgements. Image analysis was carried out at Alembic, a microscopy laboratory established by the San Raffaele Scientific Institute and Vita-Salute San Raffele University. The authors are grateful to Carla Panzeri and Alessio Palini, San Raffaele Scientific Institute, for their help with electron microscopy and cytofluorimetric analysis, respectively. We are sincerely grateful to Matthias Kieslinger (Munich) for the gift of mouse Ebf-specific shRNA vectors and Ebf2 expression vectors. GGC was supported by an Ataxia-UK grant. Additional support to this work came from Fondazione Mariani (Milan), the Italian Ministry of Health and the Canadian Institutes of Health Research.

1. Lamborghini JE. Disappearance of Rohon-Beard neurons from the spinal cord of larval Xenopus laevis. J Comp Neurol 1987; 264: 47-55.

2. Cecconi F, Alvarez-Bolado G, Meyer BI, Roth KA, Gruss P. Apaf1 (CED-4 homolog) regulates programmed cell death in mammalian development. Cell 1998; 94: 727-737.

3. Zanjani HS, Vogel MW, Delhaye-Bouchaud N, Martinou JC, Mariani J. Increased cerebellar Purkinje cell numbers in mice overexpressing a human bcl-2 transgene. J Comp Neurol 1996; 374: 332-341.
4. Huang EJ, Reichardt LF. Neurotrophins: roles in neuronal development and function. Annu Rev Neurosci 2001; 24: 677-736.

5. Dusart I, Guenet JL, Sotelo C. Purkinje cell death: differences between developmental cell death and neurodegenerative death in mutant mice. Cerebellum 2006; 5 : 163-173.

6. Doughty ML, De Jager PL, Korsmeyer SJ, Heintz N. Neurodegeneration in Lurcher mice occurs via multiple cell death pathways. J Neurosci 2000; 20: 3687-3694.

7. Dubois L, Vincent A. The COE-Collier/Olf1/EBF-transcription factors: structural conservation and diversity of developmental functions. Mech Dev 2001; 108: 3-12.

8. Pozzoli O, Bosetti A, Croci L, Consalez GG, Vetter ML. Xebf3 is a regulator of neuronal differentiation during primary neurogenesis in Xenopus. Dev Biol 2001; 233: 495-512.

9. Garel S, Marin F, Grosscheld R, Charnay P. Ebf1 controls early cell differentiation in the embryonic striatum. Development 1999; 126: 5285-5294.

10. Corradi A, Croci L, Broccoli V, Zecchini S, Previtali S, Wurst W et al. Hypogonadotropic hypogonadism and peripheral neuropathy in Ebf2-null mice. Development 2003; 130: 401-410.

11. Wang SS, Lewcock JW, Feinstein P, Mombaerts $P$, Reed RR. Genetic disruptions of O/E2 and $\mathrm{O} / \mathrm{E} 3$ genes reveal involvement in olfactory receptor neuron projection. Development 2004; 131: 1377-1388.

12. Croci L, Chung SH, Masserdotti G, Gianola S, Bizzoca A, Gennarini G et al. A key role for the HLH transcription factor EBF2COE2,O/E-3 in Purkinje neuron migration and cerebellar cortical topography. Development 2006; 133: 2719-2729.

13. Chung S-H, Marzban H, Croci L, Consalez G, Hawkes R. Purkinje cell subtype specification in the cerebellar cortex: Ebf2 acts to repress the Zebrin II-positive Purkinje cell phenotype. Neuroscience 2008; 153: 721-732.

14. D'Ercole AJ, Ye P, Calikoglu AS, Gutierrez-Ospina G. The role of the insulin-like growth factors in the central nervous system. Mol Neurobiol 1996; 13: 227-255.

15. Torres-Aleman I, Pons S, Arevalo MA. The insulin-like growth factor I system in the rat cerebellum: developmental regulation and role in neuronal survival and differentiation. J Neurosci Res 1994; 39: 117-126.

16. Chrysis D, Calikoglu AS, Ye P, $D^{\prime}$ Ercole AJ. Insulin-like growth factor-I overexpression attenuates cerebellar apoptosis by altering the expression of $\mathrm{Bcl}$ family proteins in a developmentally specific manner. J Neurosci 2001; 21: 1481-1489.

17. Carletti B, Rossi F. Neurogenesis in the Cerebellum. Neuroscientist 2007; 14: 91-100.

18. Malgaretti N, Pozzoli O, Bosetti A, Corradi A, Ciarmatori S, Panigada M et al. Mmot1, a new helix-loop-helix transcription factor gene displaying a sharp expression boundary in the embryonic mouse brain. J Biol Chem 1997; 272: 17632-17639.

19. Kudrycki K, Stein-Izsak C, Behn C, Grillo M, Akeson R, Margolis FL et al. Olf-1-binding site: characterization of an olfactory neuron-specific promoter motif. Mol Cell Biol 1993; 13: 3002-3014.

20. Lavorgna G, Guffanti A, Borsani G, Ballabio A, Boncinelli E. Targetfinder: to search annotated sequence databases for target genes of transcription factors. Bioinformatics 1999; 15: 172-173.

21. Hall LJ, Kajimoto Y, Bichell D, Kim SW, James PL, Counts D et al. Functional analysis of the rat insulin-like growth factor I gene and identification of an IGF-I gene promoter. DNA Cell Biol 1992; 11: 301-313.

22. Woelfle $J$, Billiard J, Rotwein P. Acute control of insulin-like growth factor-l gene transcription by growth hormone through Stat5b. J Biol Chem 2003; 278: 22696-22702.

23. Musaro A, Dobrowolny G, Rosenthal N. The neuroprotective effects of a locally acting IGF-1 isoform. Exp Gerontol 2007; 42: 76-80.

24. Favelyukis S, Till JH, Hubbard SR, Miller WT. Structure and autoregulation of the insulinlike growth factor 1 receptor kinase. Nat Struct Biol 2001; 8: 1058-1063.

25. Fukudome Y, Tabata T, Miyoshi T, Haruki S, Araishi K, Sawada S et al. Insulin-like growth factor-I as a promoting factor for cerebellar Purkinje cell development. Eur J Neurosci 2003; 17: 2006-2016.

26. Bondy CA, Cheng CM. Signaling by insulin-like growth factor 1 in brain. Eur J Pharmacol 2004; 490: 25-31.

27. Hetman M, Cavanaugh JE, Kimelman D, Xia Z. Role of glycogen synthase kinase-3beta in neuronal apoptosis induced by trophic withdrawal. J Neurosci 2000; 20: 2567-2574.

28. Cheng CM, Reinhardt RR, Lee WH, Joncas G, Patel SC, Bondy CA. Insulin-like growth factor 1 regulates developing brain glucose metabolism. Proc Natl Acad Sci U S A 2000; 97: 10236-10241.

29. Ozdinler PH, Macklis JD. IGF-I specifically enhances axon outgrowth of corticospinal motor neurons. Nat Neurosci 2006; 9: 1371-1381.

30. Kim BJ, Takamoto N, Yan J, Tsai SY, Tsai MJ. Chicken Ovalbumin Upstream PromoterTranscription Factor II (COUP-TFII) regulates growth and patterning of the postnatal mouse cerebellum. Dev Biol 2008; 326: 378-391.

31. Bitoun E, Finelli MJ, Oliver PL, Lee S, Davies KE. AF4 is a critical regulator of the IGF-1 signaling pathway during Purkinje cell development. $J$ Neurosci 2009; 29: 15366-15374.

32. Armengol JA, Sotelo C. Early dendritic development of Purkinje cells in the rat cerebellum. A light and electron microscopic study using axonal tracing in 'in vitro' slices. Brain Res Dev Brain Res 1991; 64: 95-114. 
33. Gardette R, Debono M, Dupont JL, Crepel F. Electrophysiological studies on the postnatal development of intracerebellar nuclei neurons in rat cerebellar slices maintained in vitro. II. Membrane conductances. Brain Res 1985; 352: 97-106.

34. Dusart I, Airaksinen MS, Sotelo C. Purkinje cell survival and axonal regeneration are age dependent: an in vitro study. J Neurosci 1997; 17: 3710-3726.

35. Hawkes R. Antigenic markers of cerebellar modules in the adult mouse. Biochem Soc Trans 1992; 20: 391-395.

36. Sarna JR, Hawkes R. Patterned Purkinje cell death in the cerebellum. Prog Neurobiol 2003; 70: 473-507.
37. Backstrom M, Hall K, Sara V. Somatomedin levels in cerebrospinal fluid from adults with pituitary disorders. Acta endocrinologica 1984; 107: 171-178.

38. Miller BH, Gore AC. Alterations in hypothalamic insulin-like growth factor-I and its associations with gonadotropin releasing hormone neurones during reproductive development and ageing. J Neuroendocrinol 2001; 13: 728-736.

39. Russell JW, Cheng HL, Golovoy D. Insulin-like growth factor-I promotes myelination of peripheral sensory axons. J Neuropathol Exp Neurol 2000; 59: 575-584.

40. Cohen $E$, Dillin A. The insulin paradox: aging, proteotoxicity and neurodegeneration. Nat Rev Neurosci 2008; 9: 759-767.

Supplementary Information accompanies the paper on Cell Death and Differentiation website (http://www.nature.com/cdd) 